PITHA-08/16

SFB/CPP-08-41

\title{
Hypergeometric functions with rational arguments
}

\author{
T. Huber ${ }^{\mathrm{a} *}$

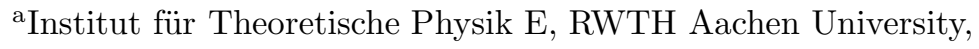 \\ D-52056 Aachen, Germany
}

We elaborate on the expansion of hypergeometric functions ${ }_{P} F_{P-1}$ about rational parameters, where we focus mainly on the integer and half-integer case. The strategy and the basic steps of a recently developed algorithm for the expansion about half-integer parameters are described. The algorithm is implemented in the Mathematica package HypExp, by means of which we derive (partially new) results of selected multi-loop Feynman diagrams. Moreover, we give a new formulation of a conjecture in the context of a three-loop master integral in HQET.

\section{INTRODUCTION}

Hypergeometric functions (HFs) have a long history in science. Their trail of success already started in the 17th and 18th century when former days' scientists like Wallis, Leibniz, Newton, Stirling, and Euler discovered hypergeometriclike patterns in sequences of numbers, mostly in the context of geometrical problems. In 1797, Pfaff and Gauß observed that the second order differential equation

$$
\begin{array}{r}
x(1-x) y^{\prime \prime}(x)+[c-(a+b+1) x] y^{\prime}(x) \\
-a b y(x)=0
\end{array}
$$

is solved by

$$
\begin{aligned}
& y(x)=A_{2} F_{1}(a, b ; c ; x) \\
& +B x_{2}^{1-c} F_{1}(1+a-c, 1+b-c ; 2-c ; x),
\end{aligned}
$$

where

${ }_{2} F_{1}(a, b ; c ; x)=\sum_{i=0}^{\infty} \frac{\Gamma(a+i) \Gamma(b+i) \Gamma(c)}{\Gamma(a) \Gamma(b) \Gamma(c+i)} \frac{x^{i}}{i !}$

represents the hypergeometric series. Later on in the 19th century, argument transformations for the ${ }_{2} F_{1}$-function were derived (Kummer), and generalizations of the series to other ${ }_{p} F_{q}$ and to two arguments (Appell, Schwarz, Riemann,

\footnotetext{
*Work supported by Deutsche Forschungsgemeinschaft,
} SFB/TR 9.
Kampé de Fériet) were developed. Nowadays, HFs have a wide range of applications in physics, mathematics, engeneering, and economics. In the field of particle physics HFs appear in loop and phase space integrals in the context of dimensional regularization, where usually the regularization parameter $\epsilon$ appears in the parameters, whereas masses and kinematic invariants constitute the argument of the HF. Due to the need of extracting poles, finite parts and higher orders in the $\epsilon$-expansion in order to ultimately make predictions for physical observables, one is oftentimes confronted with the task of expanding HFs about their parameters.

Systematic approaches to the expansion of HFs about integer-valued parameters have been developed [1-3] and have been implemented in GiNaC [4,5], Mathematica [6], and FORM [7].

In computations involving massive particles [8$20]$ the HFs can contain half-integer parameters. Methods have been developed to expand HFs with half-integer parameters [2,21-23]. Recently, we implemented a new algorithm for the expansion about half-integer parameters in the existing Mathematica package HypExp [6,24]. In the following we briefly describe this algorithm and give examples and applications of its usage. We conclude with a brief summary on the expansion of HFs about other rational parameters. 


\section{ALGORITHM}

We start the explanation of our algorithm with a definition. A HF ${ }_{P} F_{P-1}\left(\left\{A_{i}\right\} ;\left\{B_{j}\right\} ; x\right)$ is said to be of type $P_{s}^{r}$ if, at $\epsilon=0, r$ out of the $A_{i}$ and $s$ out of the $B_{j}$ are half-integers and all the others are integers. The algorithm, which was presented for the first time in Ref. [24] consists of three parts. i) Reduction. We express a HF of a given type in terms of integration and differentiation operators acting on one specific $\mathrm{HF}$ of the same type, the latter we call the basis function of this type, see Eq. (12). ii) Expansion of the basis function in $\epsilon$. The choice of the basis function for each type is not unique and we choose it such that its expansion in $\epsilon$ can be performed as conveniently as possible. iii) Application of the integration and differentiation operators. In the last step we have to find integration and differentiation routines which act on the expanded basis function.

Below, we cover each of these steps in turn. Other, related algorithms which are valid for the integer and/or half-integer case rely on the reduction to a set of basis functions by means of recurrence relations $[6,21-23]$ or on the nested (harmonic and binomial) sums approach $[1,2,25-27]$.

\subsection{Reduction}

We start the reduction part of the algorithm by introducting a few more definitions. We define the short-hand notation

$$
\begin{aligned}
\prod_{j}^{a: a}=1, \quad \prod_{j}^{a: b} f(j) & =\prod_{j=a}^{b-1} f(j) \quad \text { if } \quad a<b, \\
\prod_{j}^{a: b} f(j) & =\prod_{j=b}^{a-1} \frac{1}{f(j)} \quad \text { if } \quad a>b,
\end{aligned}
$$

so that

$$
\Gamma(b)=\Gamma(a) \prod_{j}^{a: b}(j) \quad \text { if } \quad a-b \in \mathbb{Z} .
$$

Furthermore, we define integration and differentiation operators $[1,2]$

$$
\begin{aligned}
J^{+}(j, 1)[f](x) & \equiv \frac{1}{x^{j}} \int_{0}^{x} \mathrm{~d} x^{\prime} x^{\prime j-1} f\left(x^{\prime}\right), \\
J^{-}(j, 1)[f](x) & \equiv \frac{1}{x^{j-1}} \frac{\mathrm{d}}{\mathrm{d} x} x^{j} f(x),
\end{aligned}
$$

$J^{ \pm}(j, n)[f](x) \equiv\left[J^{ \pm}(j, 1)\right]\left[J^{ \pm}(j, n-1)[f]\right](x)$,

so that

$$
\begin{aligned}
\frac{x^{i}}{(i+j)^{n}} & =J^{+}(j, n)\left[y^{i}\right](x) \quad \text { and } \\
i^{n} x^{i} & =J^{-}(0, n)\left[y^{i}\right](x) .
\end{aligned}
$$

We now consider a HF of type $2_{1}^{1}$ and start from

$$
\begin{aligned}
& { }_{2} F_{1}\left(A_{1}, A_{2} ; B_{1} ; x\right)=1+ \\
& \frac{\Gamma\left(B_{1}\right)}{\Gamma\left(A_{1}\right) \Gamma\left(A_{2}\right)} \sum_{i=1}^{\infty} \frac{\Gamma\left(A_{1}+i\right) \Gamma\left(A_{2}+i\right)}{\Gamma\left(B_{1}+i\right) \Gamma(i+1)} x^{i}
\end{aligned}
$$

with

$A_{1}=a_{1}+\frac{1}{2}+\alpha_{1} \epsilon, \quad A_{2}=a_{2}+\alpha_{2} \epsilon$,

$B_{1}=b_{1}+\frac{1}{2}+\beta_{1} \epsilon$.

We transform the $\Gamma$-functions in Eq. (8) by means of Eq. (5) and arrive at

$$
\begin{gathered}
{ }_{2} F_{1}\left(a_{1}+\frac{1}{2}+\alpha_{1} \epsilon, a_{2}+\alpha_{2} \epsilon ; b_{1}+\frac{1}{2}+\beta_{1} \epsilon ; x\right)=1+ \\
\frac{\Gamma\left(\frac{1}{2}+\beta_{1} \epsilon\right)}{\Gamma\left(\frac{1}{2}+\alpha_{1} \epsilon\right) \Gamma\left(1+\alpha_{2} \epsilon\right)} \frac{\prod_{j}^{0: b_{1}}\left(j+\frac{1}{2}+\beta_{1} \epsilon\right)}{\prod_{j}^{1}\left(j+\frac{1}{2}+\alpha_{1} \epsilon\right) \prod_{j}^{1: a_{2}}\left(j+\alpha_{2} \epsilon\right)} \\
\times \sum_{i=1}^{\infty} \underbrace{\frac{\prod_{j}^{0: a_{1}}\left(i+j+\frac{1}{2}+\alpha_{1} \epsilon\right) \prod_{j}^{1: a_{2}}\left(i+j+\alpha_{2} \epsilon\right)}{\prod_{j}^{0: b_{1}}\left(i+j+\frac{1}{2}+\beta_{1} \epsilon\right)}}_{D} \\
\times \frac{\Gamma\left(i+\frac{1}{2}+\alpha_{1} \epsilon\right) \Gamma\left(i+1+\alpha_{2} \epsilon\right)}{\Gamma\left(i+\frac{1}{2}+\beta_{1} \epsilon\right) \Gamma(i+1)} x^{i} .
\end{gathered}
$$

We now decompose of $D$ into partial fractions with respect to $i$ and obtain

$$
\begin{aligned}
D= & \sum_{j \geq 0, n} \frac{C_{j, n}^{+}}{(i+j+\gamma \epsilon)^{n}}+\sum_{j<0, n} \frac{C_{j, n}^{+}}{(i+j+\gamma \epsilon)^{n}} \\
& +\sum_{j, n} \frac{C_{j, n}^{1 / 2}}{\left(i+\frac{1}{2}+j+\gamma \epsilon\right)^{n}}+\sum_{n} C_{n}^{-} i^{n},(11)
\end{aligned}
$$

where $C_{j, n}^{+}, C_{n}^{-}$and $C_{j, n}^{1 / 2}$ are polynomials in $\epsilon$. In the first and third sum in Eq. (11) we expand 
the denominator in $\epsilon$ and write the resulting expression in terms of $J^{+}(j, n)$ and $J^{+}\left(j+\frac{1}{2}, n\right)$. In the last sum we express $i^{n} x^{i}$ in terms of $J^{-}(0, n)$. The second sum is conceptually also straightforward but results in quite lengthy formulas which we omit here. We refer the reader to Ref. [24] for details on this point.

The final formula reads

$$
\begin{aligned}
& { }_{2} F_{1}\left(a_{1}+\frac{1}{2}+\alpha_{1} \epsilon, a_{2}+\alpha_{2} \epsilon ; b_{1}+\frac{1}{2}+\beta_{1} \epsilon ; x\right)= \\
& 1+\frac{\prod_{j}^{0: b_{1}}\left(j+\frac{1}{2}+\beta_{1} \epsilon\right)}{\prod_{j}^{0: a_{1}}\left(j+\frac{1}{2}+\alpha_{1} \epsilon\right) \prod_{j}^{1: a_{2}}\left(j+\alpha_{2} \epsilon\right)} \\
& \times\left[\sum_{j \geq 0, n} \tilde{C}_{j, n}^{+} J^{+}(j, n)+\sum_{j<0, n, \gamma} \tilde{C}_{j, n, \gamma}^{+} J^{+}(j, n, \gamma)\right. \\
& \left.+\sum_{j, n} \tilde{C}_{j, n}^{1 / 2} J^{+}\left(j+\frac{1}{2}, n\right)+\sum_{n} C_{n}^{-} J^{-}(0, n)\right] B, \quad(12)
\end{aligned}
$$

with the basis function $B$ of this type,

$$
B={ }_{2} F_{1}\left(\frac{1}{2}+\alpha_{1} \epsilon, 1+\alpha_{2} \epsilon, \frac{1}{2}+\beta_{1} \epsilon, x\right)-1 .
$$

Eq. (12) is most useful at the level of the expansion in $\epsilon$ since the various $\tilde{C}$ and later on also $B$ enter this equation as expanded quantities. The generalization of this part of the algorithm to other types $2_{s}^{r}$ and to ${ }_{P} F_{P-1}$ with $P>2$ is straightforward. Moreover, this part of the algorithm is universal, i. e. type independent.

\subsection{Expansion of the basis function}

Here we make the ansatz

$$
\begin{aligned}
B= & g(x)\left[1+\sum_{j=1}^{\infty} \epsilon^{j}\right. \\
& \left.\times \sum_{\substack{s_{1}, \ldots, s_{j}=\\
+, 0,-}} c\left(s_{1}, \ldots, s_{j} ; x\right) H_{s_{1}, \ldots, s_{j}}(f(x))\right]
\end{aligned}
$$

with $f(x)=\sqrt{x}$ for HFs of type $P_{i}^{i}$ and

$f(x)=i \sqrt{\frac{x}{1-x}} \quad$ or $\quad f(x)=\frac{1-\sqrt{1-x}}{1+\sqrt{1-x}}$

for HFs of type $P_{i \pm 1}^{i}[13,16,21-23] . \quad H$ denotes a harmonic polylogarithm (HPL) [28], and the weights "+" and "-" are, respectively, the sum and the difference of the ordinary integer weights \pm 1 [29]. The function $g(x)$ is given by the value of the HF with the expansion parameter $\epsilon$ put to zero. The coefficients $c\left(s_{1}, \ldots, s_{j} ; x\right)$ have the following properties. They are homogeneous of order $j$ in the $\alpha_{i}, \beta_{i}$; and symmetric in all $\alpha$ and $\beta$ parameters which correspond to equal $a$ and $b$ parameters. Moreover, they must reduce to the coefficient of a reduced $\mathrm{HF}$ in the limit as one of the $A_{i}$ becomes equal to one of the $B_{k}$. We also make an ansatz for the $x$-dependence of $c\left(s_{1}, \ldots, s_{j} ; x\right)$. This ansatz depends on $f(x)$ and is rather simple. For type $2_{1}^{1}$ it is for instance a constant or $\sqrt{x}$ times a constant, depending on whether we have an even or odd number of "+" weights in $\left\{s_{1}, \ldots, s_{j}\right\}$. We then insert the complete ansatz for $B$ into the differential equation for the $\mathrm{HF}_{P} F_{P-1}$

$\mathcal{D} B=0$

This yields after possible variable changes

$$
\sum_{j=0}^{\infty} \epsilon^{j} \sum_{\substack{l \\ s_{1}, \ldots, s_{l}=\\+, 0,-}} \mathcal{C}\left(s_{1}, \ldots, s_{l}\right) H_{s_{1}, \ldots, s_{l}}(y)=0
$$

The differential equation is satisfied if and only if all the coefficients $\mathcal{C}\left(s_{i}\right)$ vanish. The coefficients $c\left(s_{1}, \ldots, s_{l} ; x\right)$ can be extracted from these conditions. This part of the algorithm is a case-by-case approach, i. e. the expansion of the basis function must be derived separately for each type $P_{s}^{r}$.

\subsection{Application of operators}

We are now left with the task of carrying out explicitly the integration and differentiation operations which now act on the expanded basis function. Since the HPLs which occur in the expansion of the latter are iterated integrations over rational functions they are well-suited for carrying out all required operations. The difficulties are to ensure the cancellation of all divergences $\propto 1 / x^{k}$ at the lower integration limit, the integration of structures like $1 / \sqrt{x} \cdot H[\{\ldots\}, f(x)]$, as well as the need for introducing two new weights $w_{1}(t)=1 / \sqrt{1-t^{2}}$ and $w_{2}(t)=1 /\left(t \sqrt{1-t^{2}}\right)$ whose contributions cancel in the end. 


\section{EXAMPLES}

We implemented the above algorithm in the Mathematica package HypExp $[6,24]$. The package $^{2}$ allows to expand arbitrary HFs ${ }_{P} F_{P-1}\left(\left\{A_{i}\right\} ;\left\{B_{j}\right\} ; x\right)$ about integer parameters to arbitrary order in $\epsilon$, both for general argument $x$ and for unit argument. The extension to halfinteger parameters allows the expansion of HFs of types

$2_{1}^{2}, \quad 2_{1}^{1}, \quad 2_{0}^{1}, \quad 2_{1}^{0}, \quad 3_{2}^{3}, \quad 3_{2}^{2}, \quad 3_{1}^{1}, \quad 3_{0}^{1}, \quad 3_{1}^{0}, 4_{1}^{1}, 4_{3}^{3}$

also to arbitrary power in $\epsilon$, again for both general argument $x$ and $x=1$. In the following we give examples of multi-loop diagrams which can be expanded by means of the package.

\subsection{Two-loop massive self-energy}

Our first example is the two-loop massive selfenergy diagram depicted on the left in Fig. 1. It reads

$$
\begin{aligned}
I= & \int \frac{d^{D} k_{1}}{(2 \pi)^{D}} \int \frac{d^{D} k_{2}}{(2 \pi)^{D}} \frac{1}{\left[k_{2}^{2}-M^{2}\right]} \\
& \times \frac{1}{\left[\left(k_{1}-k_{2}\right)^{2}-M^{2}\right]\left[\left(k_{1}-p_{m}\right)^{2}-m^{2}\right]},
\end{aligned}
$$

where an implicit $+i \eta(\eta>0)$ is tacitly understood. This integral can be written in terms of HFs and assumes the very simple form

$$
\begin{aligned}
I= & -S_{\Gamma}^{2}\left[M^{2}\right]^{1-2 \epsilon} \frac{\Gamma^{2}(1-\epsilon) \Gamma^{2}(\epsilon)}{(1-\epsilon)} \\
\times & \left\{\frac{1}{1-2 \epsilon}{ }_{3} F_{2}\left(\frac{1}{2}, 1,-1+2 \epsilon ; \frac{1}{2}+\epsilon, 2-\epsilon ; r\right)\right. \\
& \left.+r^{1-\epsilon}{ }_{3} F_{2}\left(1, \epsilon, \frac{3}{2}-\epsilon ; \frac{3}{2}, 3-2 \epsilon ; r\right)\right\},(19)
\end{aligned}
$$

with $S_{\Gamma}=1 /(4 \pi)^{D / 2} / \Gamma(1-\epsilon)$ and $r=\left(m^{2}-\right.$ in) $/ M^{2}$. Expanding in $\epsilon$ we find

$$
\begin{aligned}
I & =-S_{\Gamma}^{2}\left[M^{2}\right]^{1-2 \epsilon}\left\{\left(1+\frac{r}{2}\right) \frac{1}{\epsilon^{2}}+\left(3+\frac{5}{4} r-r \ln r\right) \frac{1}{\epsilon}\right. \\
+ & \left(\frac{r}{2} \ln ^{2} r-\frac{(1-r)^{2}}{r} \operatorname{Li}_{2}(1-r)+\left(1-\frac{5}{2} r\right) \ln r\right. \\
& \left.\left.+\frac{\pi^{2}}{3} r+\frac{\pi^{2}}{6 r}+6+\frac{11}{8} r\right)+\mathcal{O}(\epsilon)\right\},
\end{aligned}
$$

${ }^{2}$ The package is publicly available at http://www-theorie.physik.uzh.ch/ maitreda/HypExp/ in agreement with Ref. [30]. For $m>M$ we find an expression for $I$ in terms of $\tilde{r}=\left(M^{2}-i \eta\right) / m^{2}$,

$$
\begin{aligned}
& I=S_{\Gamma}^{2}\left[m^{2}\right]^{1-2 \epsilon} 2^{1-2 \epsilon} \Gamma^{2}(1-\epsilon) \\
& \times\left\{\frac{\Gamma^{2}(\epsilon) \tilde{r}^{1-\epsilon} 2^{2 \epsilon}}{(\epsilon-1)(1-2 \epsilon)}{ }_{3} F_{2}\left(\frac{1}{2}, 1,2 \epsilon-1 ; \frac{1}{2}+\epsilon, 2-\epsilon ; \tilde{r}\right)\right. \\
& \quad+\frac{2^{2 \epsilon} \Gamma^{2}(\epsilon) \tilde{r}^{2-2 \epsilon}}{4(\epsilon-1)}{ }_{3} F_{2}\left(1, \frac{3}{2}-\epsilon, \epsilon ; \frac{3}{2}, 3-2 \epsilon ; \tilde{r}\right) \\
& +\frac{\pi \Gamma\left(-\frac{1}{2}+\epsilon\right) \Gamma\left(-\frac{3}{2}+2 \epsilon\right) \tilde{r}^{\frac{3}{2}-2 \epsilon}}{\Gamma(\epsilon)} \\
& \quad+\frac{2^{1-2 \epsilon} \Gamma^{2}(1-\epsilon) \Gamma(\epsilon) \Gamma\left(\frac{3}{2}-2 \epsilon\right) \Gamma(-1+2 \epsilon)}{\sqrt{\pi} \Gamma(3-3 \epsilon)} \\
& \left.\quad \times{ }_{2} F_{1}\left(-2+3 \epsilon,-\frac{1}{2}+\epsilon ;-\frac{1}{2}+2 \epsilon ; \tilde{r}\right)\right\} .(21)
\end{aligned}
$$

Upon expansion in $\epsilon$ we find

$$
\begin{aligned}
& I=-S_{\Gamma}^{2}\left[m^{2}\right]^{1-2 \epsilon}\left\{\left(\frac{1}{2}+\tilde{r}\right) \frac{1}{\epsilon^{2}}+\left(\frac{5}{4}+3 \tilde{r}-2 \tilde{r} \ln \tilde{r}\right) \frac{1}{\epsilon}\right. \\
& +\left(\frac{\tilde{r}}{2}(2+\tilde{r}) \ln ^{2} \tilde{r}+(1-\tilde{r})^{2} \operatorname{Li}_{2}(1-\tilde{r})-7 \tilde{r} \ln \tilde{r}\right. \\
& \left.\left.+\frac{\pi^{2}}{3}+\frac{\pi^{2}}{6} \tilde{r}^{2}+6 \tilde{r}+\frac{11}{8}\right)+\mathcal{O}(\epsilon)\right\} .
\end{aligned}
$$

This result can also be obtained by analytic continuation of Eq. (20) and proper inclusion of the analytic continuation sign.

\subsection{Three-loop HQET master integral}

We elaborate only briefly on this integral since it has been discussed at length in Ref. [19]. It can be written as

$$
\begin{aligned}
I_{n_{1} n_{2} n_{3}}= & \frac{1}{i \pi^{d / 2}} \int \frac{I_{n_{1} n_{2}}^{2}\left(p_{0}\right) d^{d} p}{\left(1-p^{2}-i 0\right)^{n_{3}}}, \\
I_{n_{1} n_{2}}\left(p_{0}\right)= & \frac{1}{i \pi^{d / 2}} \int \frac{d^{d} k}{\left(-2\left(k_{0}+p_{0}\right)-i 0\right)^{n_{1}}} \\
& \times \frac{1}{\left(1-k^{2}-i 0\right)^{n_{2}}},
\end{aligned}
$$

and a closed form in terms of HFs with halfinteger parameters was given in Ref. [19]. We focus here on a particular combination of indices, namely

$\frac{I_{122}}{\Gamma^{3}(1+\epsilon)}=\frac{1}{2 \epsilon^{2}}\left[\frac{2 \Gamma^{2}(1-\epsilon) \Gamma^{3}(1+2 \epsilon)}{\Gamma^{2}(1+\epsilon) \Gamma(1-2 \epsilon) \Gamma(2+4 \epsilon)}\right.$ 

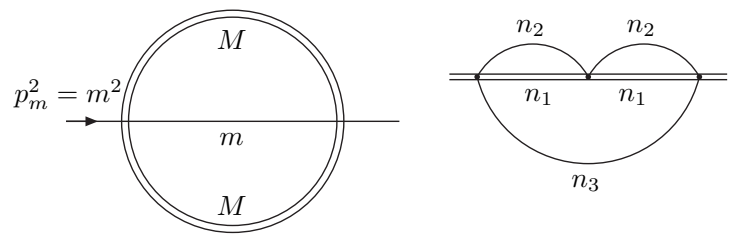

Figure 1. Left panel: Two-loop massive self energy. Right panel: Three-loop on-shell HQET propagator diagram with mass.

$$
\begin{array}{r}
\times{ }_{3} F_{2}\left(\begin{array}{c}
\frac{1}{2}, 1+2 \epsilon,-\epsilon \\
\frac{3}{2}+2 \epsilon, 1-\epsilon
\end{array} \mid 1\right) \\
-\frac{1}{1+2 \epsilon}{ }_{4} F_{3}\left(\begin{array}{c}
1, \frac{1}{2}-\epsilon, 1+\epsilon,-2 \epsilon \\
\frac{3}{2}+\epsilon, 1-\epsilon, 1-2 \epsilon
\end{array} \mid 1\right) \\
\left.-\frac{\Gamma^{2}(1-\epsilon) \Gamma^{4}(1+2 \epsilon) \Gamma(1-2 \epsilon) \Gamma^{2}(1+3 \epsilon)}{\Gamma^{4}(1+\epsilon) \Gamma(1+4 \epsilon) \Gamma(1-4 \epsilon) \Gamma(2+6 \epsilon)}\right] .
\end{array}
$$

In Ref. [19] we formulated the conjecture that the above expression is equal to

$$
\frac{I_{122}}{\Gamma^{3}(1+\epsilon)}=\frac{\pi^{2}}{3} \frac{\Gamma^{3}(1+2 \epsilon) \Gamma^{2}(1+3 \epsilon)}{\Gamma^{6}(1+\epsilon) \Gamma(2+6 \epsilon)} .
$$

The conjecture was formulated based on the agreement of the expansions up to the seventh order in $\epsilon$. Moreover, we performed further numerical checks at the level of the unexpanded expressions for various values of $\epsilon$ on the real axis and in the complex plane. In Ref. [31] a reformulation of the above conjecture was given. Here we give another, alternative and very simple reformulation of the conjecture.

$$
\begin{aligned}
& G_{44}^{33}\left(1 \mid \begin{array}{c}
\left\{0,-\epsilon, \frac{1}{2}+\epsilon\right\},\{1+2 \epsilon\} \\
\{0, \epsilon, 2 \epsilon\},\left\{-\frac{1}{2}-\epsilon\right\}
\end{array}\right) \\
& =\frac{2^{2+4 \epsilon} \pi^{2} \Gamma(1-2 \epsilon) \Gamma^{2}(1+2 \epsilon) \Gamma^{2}(1+3 \epsilon)}{3 \Gamma(1-\epsilon) \Gamma^{3}(1+\epsilon) \Gamma(2+6 \epsilon)} .
\end{aligned}
$$

Again, we have strong numerical evidence for this conjecture to hold true for any $\epsilon$ but we still lack an analytic proof of the collaps of the MeijerGfunction to mere $\Gamma$-functions.

\subsection{Three-loop master integral $A_{6,2}$}

Our third example is the three-loop master integral $A_{6,2}$ which is displayed on the left in Fig. 2. It was calculated in Refs. [24,32] using two different methods, one based on HFs, the other one based on a two-dimensional Mellin-Barnes representation. Following the former method, we have

$$
\begin{aligned}
& A_{6,2}=\int \frac{d^{D} k}{(2 \pi)^{D}} \int \frac{d^{D} l}{(2 \pi)^{D}} \int \frac{d^{D} r}{(2 \pi)^{D}} \frac{1}{\left(k+p_{1}\right)^{2}} \\
& \times \frac{1}{\left(k+l-p_{2}\right)^{2} l^{2} r^{2}(r-k)^{2}(r-k-l)^{2}} \\
&=\frac{\mathcal{N} 2^{8 \epsilon-2} \pi \Gamma^{2}(1-3 \epsilon) \Gamma^{5}(1-\epsilon) \Gamma(3 \epsilon)}{\epsilon \Gamma(2-4 \epsilon) \Gamma^{2}\left(\frac{3}{2}-2 \epsilon\right)} \\
& \times \int_{0}^{1} d s s^{\epsilon-1} \bar{s}^{-3 \epsilon}\left[\frac{s^{\epsilon} \Gamma(1-\epsilon)^{2}}{\Gamma(1-2 \epsilon)}-{ }_{2} F_{1}(\epsilon,-\epsilon ; 1-\epsilon ; s)\right. \\
& \times{ }_{3} F_{2}\left(1-3 \epsilon, 1-2 \epsilon, 1-\epsilon ; 2-4 \epsilon, \frac{3}{2}-2 \epsilon ;-\frac{\bar{s}^{2}}{4 s}\right),
\end{aligned}
$$

with $\bar{s}=1-s$ and

$\mathcal{N}=\frac{i(4 \pi)^{3 \epsilon-6}}{\Gamma^{3}(1-\epsilon)}\left(-q^{2}-i \eta\right)^{-3 \epsilon}$.

Eq. (27) can be expanded in $\epsilon$ at the level of the integrand, which yields HPLs of argument $-(1-s) /(1+s)$ that can be converted to HPLs of argument $s$ by applying (twice) the command HPLConvertToSimplerArguments from the HPL $[29,33]$ package. The next step is to expand the product of HPLs into a sum of HPLs which we can then integrate by means of the integration routines of HPL. This procedure is not restricted to a specific depth of the expansion, so we could, in principle, expand $A_{6,2}$ to all orders. We expanded $A_{6,2}$ up to transcendentality to eight,

$$
\begin{aligned}
& A_{6,2}=\frac{\mathcal{N}}{(1-5 \epsilon)(1-4 \epsilon) \epsilon}\left[-2 \zeta_{3}-\epsilon \frac{7 \pi^{4}}{180}\right. \\
& +\epsilon^{2}\left(\frac{2}{3} \pi^{2} \zeta_{3}-10 \zeta_{5}\right)+\epsilon^{3}\left(\frac{163 \pi^{6}}{7560}+76 \zeta_{3}^{2}\right) \\
& +\epsilon^{4}\left(\frac{55}{18} \pi^{4} \zeta_{3}+\frac{445 \zeta_{7}}{2}\right)+\epsilon^{5}\left(-\frac{744}{5} \zeta_{5,3}-22 \pi^{2} \zeta_{3}^{2}\right.
\end{aligned}
$$




$$
\left.\left.+1000 \zeta_{3} \zeta_{5}+\frac{802183 \pi^{8}}{4536000}\right)+\mathcal{O}\left(\epsilon^{6}\right)\right],
$$

where we have encountered a multiple zeta value in the last term.

\subsection{Four-loop tadpole with 3 massive lines}

Our last example is the four-loop tadpole with three equal massive lines displayed on the right in Fig. 2. In Ref. [34], a one-dimensional MellinBarnes representation was derived for arbitrary powers of propagators. We consider here the case of unit propagator powers and write

$$
\begin{aligned}
& T=\int\left[d k_{1}\right] \int\left[d k_{2}\right] \int\left[d k_{3}\right] \int\left[d k_{4}\right] \frac{1}{\left[k_{1}^{2}-m^{2}\right]\left[k_{3}^{2}\right]} \\
& \times \frac{1}{\left[\left(k_{1}+k_{2}\right)^{2}-m^{2}\right]\left[\left(k_{2}+k_{3}+k_{4}\right)^{2}-m^{2}\right]\left[k_{4}^{2}\right]} \\
& \quad=-\left(m^{2}\right)^{3-4 \epsilon} e^{4 \epsilon \gamma_{E}} \Gamma(1-\epsilon) /(1-\epsilon) \\
& \times \int \frac{d z}{2 \pi i} \Gamma^{2}(1-\epsilon-z) \Gamma(-z) \Gamma(2-2 \epsilon-z) \\
& \times \frac{\Gamma(-2+3 \epsilon+z) \Gamma(-3+4 \epsilon+z)}{\Gamma(2-2 \epsilon-2 z)},
\end{aligned}
$$

with $[d k]=e^{\epsilon \gamma_{E}} /\left(i \pi^{D / 2}\right) d^{D} k$ and $D=4-2 \epsilon$ as usual. Setting also the mass equal to unity and summing all residues of left poles of $\Gamma$-functions in Eq. (30), the result can be displayed in the following closed form

$$
\begin{aligned}
& T=\frac{2^{3-4 \epsilon} e^{4 \epsilon \gamma_{E}} \pi \Gamma^{2}(1-\epsilon)}{\sin (\pi \epsilon) \Gamma(2-\epsilon)} \\
& \times\left[\frac{\sqrt{\pi} \Gamma(\epsilon) \Gamma(-1+2 \epsilon) \Gamma(-2+3 \epsilon)}{\Gamma(2-\epsilon) \Gamma\left(-\frac{1}{2}+2 \epsilon\right)}\right. \\
& \quad \times{ }_{3} F_{2}\left(\epsilon, 2 \epsilon-1,3 \epsilon-2 ; 2-\epsilon,-\frac{1}{2}+2 \epsilon ; \frac{1}{4}\right) \\
& -\frac{\Gamma\left(-\frac{1}{2}+\epsilon\right) \Gamma(-2+3 \epsilon) \Gamma(-3+4 \epsilon)}{\Gamma\left(-\frac{3}{2}+3 \epsilon\right)} \\
& \left.\times{ }_{3} F_{2}\left(2 \epsilon-1,3 \epsilon-2,4 \epsilon-3 ; \epsilon,-\frac{3}{2}+3 \epsilon ; \frac{1}{4}\right)\right] .
\end{aligned}
$$

After some manipulations and simplifications on harmonic polylogarithms, one gets for the expansion in $\epsilon$ up to the finite part

$$
T=\frac{1}{4 \epsilon^{4}}+\frac{1}{\epsilon^{3}}+\left(\frac{97}{48}+\frac{\pi^{2}}{12}\right) \frac{1}{\epsilon^{2}}
$$
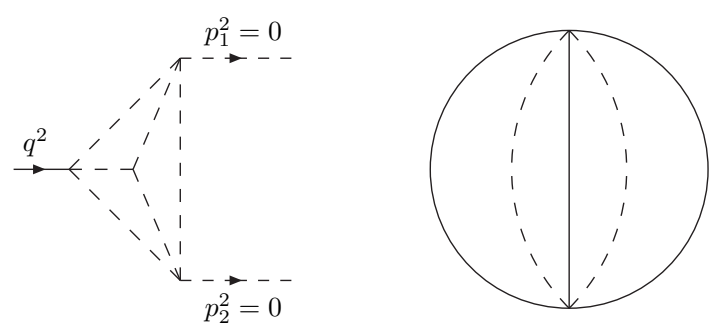

Figure 2. Left panel: Three-loop master integral $A_{6,2}$ with massless lines. Right panel: Four-loop tadpole diagram with two massless lines (dashed) and three massive ones (solid) with equal masses.

$$
\begin{aligned}
& +\left(\frac{833}{288}+\frac{\pi^{2}}{3}-\frac{\zeta_{3}}{3}\right) \frac{1}{\epsilon}+\frac{4177}{432}+\frac{97 \pi^{2}}{144}-\frac{4 \zeta_{3}}{3}+\frac{\pi^{4}}{12} \\
& +\frac{1}{1728}\left[99+16 \pi^{2}-24 \psi^{(1)}\left(\frac{1}{3}\right)\right]^{2}+\mathcal{O}(\epsilon),
\end{aligned}
$$

in agreement with the findings of Refs. [34-36].

\section{CONCLUSION AND OUTLOOK}

The field of expanding hypergeometric functions ${ }_{P} F_{P-1}$ about their parameters has acheived a quite sophisticated level. The expansion about integer parameters involves ordinary HPLs only [3], and algorithms for their all-order expansions have been developed [1-3] and implemented in computer algebra systems $[4,6,7]$.

For the expansion about half-integral parameters there exist also several algorithms [2, 21-24] for the all-order treatment of many types $P_{s}^{r}$, and also implementations in various computer languages $[4,24]$. However, not all types $P_{s}^{r}$ can be expanded entirely in terms of ordinary HPLs, counterexamples involve for instance the types $2_{0}^{2}$ and $3_{2}^{1}[24]$.

In the context of the expansion of HFs about other rational parameters some pioneering work was done by Weinzierl [2] for the cases of so-called balanced fractions

$\frac{\Gamma\left(n+a_{1}-\frac{p_{1}}{q_{1}}+b_{1} \epsilon\right)}{\Gamma\left(n+c_{1}-\frac{p_{1}}{q_{1}}+d_{1} \epsilon\right)} \frac{\Gamma\left(n+a_{2}-\frac{p_{2}}{q_{2}}+b_{2} \epsilon\right)}{\Gamma\left(n+c_{2}-\frac{p_{2}}{q_{2}}+d_{2} \epsilon\right)} \ldots$

as well as single unbalanced rational numbers in 
numerator or denominator,

$\frac{\Gamma\left(n+1-\frac{p}{q}+b \epsilon\right)}{\Gamma(n+1+d \epsilon)}$ or $\frac{\Gamma(n+a+b \epsilon)}{\Gamma\left(n+c-\frac{p}{q}+d \epsilon\right)}$.

However, up to now there are only few examples of HFs that contain other parameters than integral or half-integral ones. One important application can be found in Ref. [10].

For the algorithm described here, the extension to arbitrary rational parameters is not a problem for the reduction part. However, the expansion of the respective basis functions and the application of the differentiation and integration operators on the expanded basis functions requires more conceptual work.

\section{ACKNOWLEDGMENTS}

I would like to thank the organizers of Loops \& Legs 2008 for creating a pleasant and inspiring atmosphere. Special thanks goes to Daniel Maître for a fruitful collaboration at all stages of the HypExp project, and for a careful reading of the present manuscript.

\section{REFERENCES}

1. S. Moch, P. Uwer and S. Weinzierl, J. Math. Phys. 43, 3363 (2002)

2. S. Weinzierl, J. Math. Phys. 45 (2004) 2656

3. M. Y. Kalmykov, B. F. L. Ward and S. A. Yost, JHEP 0711, 009 (2007)

4. S. Weinzierl, Comput. Phys. Commun. 145, 357 (2002)

5. C. W. Bauer, A. Frink and R. Kreckel, arXiv:cs/0004015.

6. T. Huber and D. Maître, Comput. Phys. Commun. 175, 122 (2006)

7. S. Moch and P. Uwer, Comput. Phys. Commun. 174, 759 (2006)

8. A. I. Davydychev and J. B. Tausk, Nucl. Phys. B 397, 123 (1993).

9. D. J. Broadhurst, J. Fleischer and O. V. Tarasov, Z. Phys. C 60, 287 (1993)

10. O. V. Tarasov, Phys. Lett. B 638 (2006) 195

11. A. I. Davydychev and A. G. Grozin, Phys. Rev. D 59, 054023 (1999)
12. J. Fleischer, F. Jegerlehner and O. V. Tarasov, Nucl. Phys. B 672, 303 (2003)

13. F. Jegerlehner, M. Y. Kalmykov and O. Veretin, Nucl. Phys. B 658, 49 (2003)

14. F. Jegerlehner and M. Y. Kalmykov, Nucl. Phys. B 676, 365 (2004)

15. A. I. Davydychev and M. Y. Kalmykov, Nucl. Phys. B 605, 266 (2001)

16. A. I. Davydychev and M. Y. Kalmykov, Nucl. Phys. B 699, 3 (2004)

17. Y. Schroder and A. Vuorinen, JHEP 0506, 051 (2005)

18. E. Bejdakic and Y. Schroder, Nucl. Phys. Proc. Suppl. 160, 155 (2006)

19. A. G. Grozin, T. Huber and D. Maître, JHEP 0707, 033 (2007)

20. M. Argeri and P. Mastrolia, Int. J. Mod. Phys. A 22, 4375 (2007)

21. M. Y. Kalmykov, JHEP 0604, 056 (2006)

22. M. Y. Kalmykov, B. F. L. Ward and S. Yost, JHEP 0702, 040 (2007)

23. M. Y. Kalmykov, B. F. L. Ward and S. A. Yost, JHEP 0710, 048 (2007)

24. T. Huber and D. Maître, Comput. Phys. Commun. 178, 755 (2008)

25. J. A. M. Vermaseren, Int. J. Mod. Phys. A 14 (1999) 2037

26. A. Gonzalez-Arroyo, C. Lopez and F. J. Yndurain, Nucl. Phys. B 153 (1979) 161.

27. J. Blumlein, Comput. Phys. Commun. 159 (2004) 19

28. E. Remiddi and J. A. M. Vermaseren, Int. J. Mod. Phys. A 15 (2000) 725

29. D. Maître, arXiv:hep-ph/0703052.

30. M. Argeri, P. Mastrolia and E. Remiddi, Nucl. Phys. B 631 (2002) 388

31. A. G. Grozin, arXiv:0805.1474 [hep-ph].

32. G. Heinrich, T. Huber and D. Maître, Phys. Lett. B 662, 344 (2008)

33. D. Maître, Comput. Phys. Commun. 174, 222 (2006)

34. J. Gluza, K. Kajda and T. Riemann, Comput. Phys. Commun. 177 (2007) 879

35. R. Boughezal and M. Czakon, Nucl. Phys. B 755, 221 (2006)

36. M. Faisst, P. Maierhoefer and C. Sturm, Nucl. Phys. B 766, 246 (2007) 